Revista de Filosofía y Teoría Política, n. ${ }^{\circ}$ 48, 2017, e011, ISSN 2314-2553

Universidad Nacional de La Plata.

Facultad de Humanidades y Ciencias de la Educación.

Departamento de Filosofía

\title{
La auto-relación auténtica en $O$ lo uno o lo otro II de Kierkegaard: el amor a sí mismo como superación ética de la desesperación
}

\section{The authentic self-relation in Kierkegaard's Either Or Il: the self-love as ethic superation of despair}

\section{Pablo Uriel Rodríguez}

Universidad de Buenos Aires - Universidad de General Sarmiento, Argentina | blirius@hotmail.com.

\section{PALABRAS CLAVE}

Kierkegaard

Amor a sí mismo

Elección

Ética

\section{RESUMEN}

El artículo explora la noción de "amor propio" en Kierkegaard O lo uno o lo otro. B, el pseudónimo ético, propone no sólo un concepto negativo de "amor propio" o "egoísmo", sino también un concepto positivo de "amor propio". Sin "amor propio", la auténtica personalidad no puede desarrollarse: amarse a sí mismo significa "elegirse a uno mismo". Por "la elección de uno mismo", el individuo integra los diferentes aspectos de su personalidad en una unidad, encuentra sus propios deberes y establece las bases para una verdadera relación con el mundo y el otro.

\section{KEYWORDS}

Kierkegaard

Self-love

Choose

Ethic

\section{ABSTRACT}

The article explores the notion of "self-love" in Kierkegaard's Either / Or. B, the ethical pseudonymous, proposes not only a negative concept of "self-love" or "egoism", but also a positive concept of "self-love". Without "self love", the authentic personality can not be developed: to love oneself means "choose oneself". By "choosing oneself" the individual integrates the different aspects of his personality into a unity, finds his own duties and sets the basis for a true relationship with the world and the other. 
La noción de afirmación de sí mismo es uno de los núcleos centrales del proyecto filosófico de la Modernidad. Igualmente consustancial al pensamiento moderno es la preocupación en torno a los límites y posibles efectos negativos de esta auto-afirmación. El presente trabajo tiene como objetivo analizar el peculiar formato que adquiere este horizonte de problemas en la obra de Kierkegaard. El pensador danés se apropia del concepto de auto-afirmación en los términos de amor a sí mismo. Es de nuestro interés específico abordar el tratamiento que Kierkegaard hace de esta noción exclusivamente desde la perspectiva ética defendida en $O$ lo uno o lo otro II. Nos proponemos, por tanto, evaluar los argumentos que ofrece B a favor de la superioridad de la concepción ética de la vida por sobre la concepción estética de la vida, sin referirnos al posterior desarrollo teórico en el que comienzan a jugar categorías que, por lo menos en la comprensión que de ellas tiene el danés, exceden lo estrictamente filosófico. El texto firmado por el pseudónimo B pone de manifiesto la doble arista moderna del concepto de auto-afirmación: (1.) introduce una rehabilitación del concepto de amor a sí mismo, (2.) sólo posible desde una demarcación de sus formas defectivas. El trabajo en cuestión se articula del siguiente modo: (i.) nos enfocamos en el tratamiento que recibe el concepto de amor a sí mismo en las obras tardías de Kierkegaard (Las obras del amor y La enfermedad mortal) para llamar la atención sobre ciertos aspectos del análisis maduro de la cuestión que permiten un mejor acercamiento al primer tratamiento que recibe esta temática en la obra del danés; (ii.) realizamos una breve localización de $O$ lo uno o lo otro dentro del proyecto literario de Kierkegaard, con el fin de establecer la peculiaridad de nuestro abordaje; (iii.) nos ocupamos de las críticas que el pseudónimo ético dirige contra el modo de vida estético; (iv.) procuramos reconstruir la teoría de la auto-elección existencial en los términos de una promoción de una relación consigo mismo positiva, sobre el final de este punto ofrecemos una reformulación de la noción kierkegaardena de amor a sí mismo, a partir de la noción aristotélica de amistad consigo mismo (Ética Nicomaquea, Libro X); y (v.) hacemos lugar a unas breves consideraciones finales en las cuales dejamos planteados los límites que el propio Kierkegaard, a través de sus pseudónimos, encontraba en la propuesta de su pseudónimo ético.

\section{i. Delimitación de la problemática: el amor a sí mismo en Las obras del amor y La enfermedad mortal}

Siete años después de haber defendido su tesis doctoral sobre el concepto de estética en Kierkegaard $\stackrel{1}{ }$, Adorno pronuncia en Nueva York, ante un círculo de teólogos, una conferencia titulada "La doctrina kierkegaardiana del amor" dedicada a Las obras del amor de 1847. Con el paso del tiempo, la intervención del filósofo frankfurtiano, compartida y repudiada casi por igual, se convirtió en el peaje obligado de todos los que pretenden realizar un análisis detallado de la doctrina del amor kierkegaardiana. El breve escrito de Adorno somete la obra del danés a una aguda y contundente crítica, planteando dos objeciones fundamentales, una de índole socio-política (i.) de tendencia marxista y la otra de índole antropológica-psicológica (ii.) de tendencia psicoanalítica ${ }^{2}$ : (i.) la teoría del amor de Kierkegaard, muy lejos de ser un instrumento de combate contra las injusticias económicas, termina siendo el complemento ideológico de las inequidades materiales y simbólicas de nuestra sociedad y (ii.) la reelaboración abstracta y formalista del deber cristiano de amor al prójimo pone al individuo, cuando este se propone su cumplimiento, en contradicción con su propia naturaleza corporal (sensible) y anímica (mental). 
El interés principal de nuestra presentación nos lleva a dejar de lado el primer cuestionamiento y concentrarnos en el segundo. Lo primero que debe señalarse, en lo que respecta a la segunda objeción, es que la crítica del filósofo alemán no es gratuita y encuentra en la letra misma del escrito del danés puntos de apoyo. Adorno hace pie en numerosos pasajes del corpus kierkegaardiano que parecen plantear una oposición sin reconciliación, ni transición gradual, entre el amor cristiano y las diversas modalidades del amor mundano. Quienes emprenden la defensa de Kierkegaard, procurando contrarrestar la opinión de Adorno, centran su mirada en otros pasajes en los cuales el danés expresa que el amor al prójimo no viene a suprimir al amor mundano sino, más bien, a elevarlo por sobre sus imperfecciones: el imperativo cristiano le asigna al individuo la tarea de transfigurar religiosamente su relación con el otro para que ésta alcance el verdadero cumplimiento de sus promesas. El mandamiento cristiano de amor al prójimo es pensado como la pauta normativa que permite detectar las falencias inherentes a todos aquellos vínculos intersubjetivos que se basan en lo estrictamente humano. Sin embargo, el libro Las obras del amor añade al precepto cristiano una segunda función. La preocupación central de la obra no es tanto la de evaluar la relación entre dos sujetos, sino la de exhortar a cada uno de ellos al auto-examen, lo que quiere decir que la obra exige que cada quien se juzgue a sí mismo y evalúe de qué modo se comporta en su relación con el otro. La pregunta medular que todo cristiano debe hacerse a sí mismo en tanto amante, interrogante que resuena por detrás de cada una de las páginas del escrito kierkegaardiano, no es “la dinámica de la relación que establezco con el otro ¿es correcta?”, sino, más bien, “dentro de esta relación que he constituido con el otro, ¿̇actúo yo de modo correcto?”. De acuerdo con Kierkegaard, el individuo encuentra la guía a partir de la cual responder esta pregunta en la formulación misma del precepto cristiano. Si el individuo quiere saber si su relación con el otro está a la altura de las exigencias de un vínculo intersubjetivo auténtico, debe preguntarse “¿amo al otro como me amo a mí mismo?”: “«¿Cómo he de amar a mi prójimo?» Toda esa verborrea chocará con el precepto, parco en palabras, que no cesa de repetir imperturbablemente aquellas breves: «Como a tí mismo»” (SKS 9, p. 28 / 1965, p. 68). Uno de los aspectos más interesantes de la teoría kierkegaardiana del amor es que ella introduce un aspecto complementario que complejiza el análisis de la relación del yo con el otro. El yo debe plantearse con suma seriedad de qué modo ama al otro, pero al hacerlo no debe dejar de indagar, a la par, cómo se ama a sí mismo. Las obras del amor distingue explícitamente entre un amor a sí mismo positivo y un amor a sí mismo negativo (Cfr. SKS 9, pp. 30 - 31/ 1965, pp. 71 - 73) En el segundo tipo de amor, el lector del libro podrá rastrear la raíz profunda de todas las imperfecciones inherentes a las diversas modalidades del amor mundano; . el El primer tipo de amor, por el contrario, será concebido como uno de los pilares fundamentales del amor al prójimo. Expresado con la terminología propia de La enfermedad mortal: la relación del yo con el otro está condicionada por la relación del yo consigo mismo ${ }^{3}$; por lo tanto, cuando el yo toma como objeto de su reflexión el carácter del amor que se profesa a sí mismo, lo que hace, a su vez, es tematizar el modelo de auto-relación que él reproduce en sus distintos vínculos intersubjetivos ${ }^{4}$.

Si nos atenemos a lo que hemos dicho hasta aquí debemos concluir que las formas patológicas del amor descriptas a lo largo de los dos volúmenes de Las obras del amor son, todas ellas, indicios de una auto-relación defectiva, es decir, signos de que el individuo se ama a sí mismo de un modo impropio. Cabe notar que, en virtud del interés principal de la teoría kierkegaardiana del amor, a 
saber, el análisis del desempeño del yo en su relación con el otro, el modo en que el yo se relaciona consigo mismo se convierte en un aspecto marginal del análisis kierkegaardiano. Lo que en Las obras del amor todavía juega un papel periférico, dos años después, en La enfermedad mortal pasa a ocupar el centro de la escena. Este cambio en el foco de atención permite que el escrito pseudónimo de Anti-Climacus salde ciertas deudas del libro firmado con nombre propio. Mientras que en Las obras del amor parecería ser que el amor a sí mismo impropio era tematizado únicamente bajo la modalidad de la auto-valoración desmedida ${ }^{5}$, el análisis desarrollado en $L a$ enfermedad mortal se muestra especialmente sensible al fenómeno del menosprecio de sí. Es preciso señalar que el tratamiento de la cuestión ofrecido en La enfermedad mortal, a nuestro entender, no sólo es más completo sino que también emplea una terminología que posibilita un acercamiento más claro y preciso al concepto y la experiencia del amor a sí mismo. Al comienzo de su obra, Anti-Climacus define al yo como una actividad auto-relacional. Esta actividad de flexión sobre sí mismo es concebida como una auto-conciencia intencional de carácter volitivo. Dicho de otro modo, al tratar consigo mismo el yo no asume una actitud meramente cognoscitiva frente a su propio ser, sino que asume una actitud de implicación existencial ante su realidad, el yo no limita su actividad a la mera contemplación de sí, sino que ensaya una afirmación de sí mismo $\underline{6}$. Los distintos tipos de desesperación consciente (desesperación-debilidad y desesperación-desafío) cubren el abanico de los posibles modos negativos de la auto-relación, o sea, los modos impropios del amor a sí mismo ${ }^{\underline{7}}$ : el rechazo o la afirmación excesiva de sí ${ }^{8}$. En contraposición a la descripción de las formas incorrectas de amor a sí mismo, en La enfermedad mortal también encontramos la definición del amor a sí mismo propio que, como resulta esperable, viene a coincidir con la superación de las diversas modalidades de la desesperación $\stackrel{9}{ }$.

El cambio de perspectiva entre Las obras del amor y La enfermedad mortal implica, de alguna manera, la reanudación de una línea de reflexión abierta en El equilibrio entre lo estético y lo ético en la formación de la personalidad, el anteúltimo escrito del segundo volumen de $O$ lo uno o lo otro. Aunque reformulados, todavía es posible reconocer en el libro de 1849 los conceptos y argumentos centrales del texto de 1843. Por un lado, Anti-Climacus vuelve a utilizar la categoría de la desesperación como clave de acceso para una comprensión profunda de la realidad humana, aunque complejizando, eso sí, su función dentro de la teoría del devenir subjetivo; por otro lado, recupera la elección del sí mismo con la novedad de que ahora se trata de querer o no querer ser sí mismo ante Dios. Esta similitud temática y teórica entre ambas obras nos alienta a buscar en la segunda parte de $O$ lo uno o lo otro una conceptualización del amor a sí mismo que también distinga entre formas positivas y negativas del auto-relacionarse.

\section{ii. Una breve puntualización metodológica}

En Teoría y praxis en Las obras del amor, Patricia Dip señala que el libro titulado Las obras del amor viene a cerrar la teoría erótica kierkegaardiana iniciada en 1843 con la publicación de $O$ lo uno o lo otro. Para Dip "esto implica la comprensión de que finalmente no existe alternativa alguna entre lo estético y lo ético, sino más bien entre la desesperación que ambas esferas suponen y la fe” (2012, p. 11). En otras palabras, el que comparte el punto de vista que Kierkegaard defiende en Las obras del amor no considera que el amor-decisión del pseudónimo ético suponga una auténtica y 
definitiva corrección del déficit presente en el amor-pasión estético. Algo similar ocurre en lo que respecta al amor a sí mismo, con la salvedad de que, para nosotros, la teoría kierkegaardiana del amor propio culmina con La enfermedad mortal. En este sentido, como señala Patricia Dip en relación con el amor al otro, cuando se introduce la perspectiva religiosa la postura estética y ética quedan resignificadas. Por tanto, quien adopta el argumento de Anti-Climacus se ve forzado a considerar que la auto-relación del existente ético es insatisfactoria, como también lo es la autorelación del existente estético o, dicho en conformidad con nuestro planteo, que el amor a sí mismo ético sigue siendo tan impropio como aquel amor a sí mismo estético que la posición ética intenta superar. El trabajo que aquí emprendemos se propone resistir la tentación de leer $O$ lo uno o lo otro desde la óptica de La enfermedad mortal. Asumiendo esta perspectiva, intentamos desarrollar un análisis del primer libro pseudónimo atento a las diferencias esenciales entre la propuesta estética y la ética.

Antes de comenzar con el análisis del tratamiento ético del amor a sí mismo, es necesario plantear una cuestión preliminar: ¿es $O$ lo uno o lo otro una auténtica alternativa excluyente entre lo estético y lo ético? La solución a este interrogante no es unívoca y depende de la esfera existencial en la cual está situado quien responda. La respuesta es positiva en un caso y negativa, aunque por motivos diversos, en los dos restantes. Es positiva, desde el punto de vista del individuo estético para quien encarnar el modelo vital propuesto por el pseudónimo ético equivale a abandonar las principales características de su existencia. Es negativa, desde el punto de vista del individuo ético para quien su proyecto existencial logra integrar los aspectos positivos de lo estético en una concepción superior de la vida. También es negativa para el individuo religioso, puesto que en definitiva ambas esferas se revelan igualmente insatisfactorias en relación a la existencia cristiana $\underline{10}$.

Si se la examina desde el punto de vista religioso la existencia ética es todavía una existencia malograda. Sin embargo, este juicio implica una evaluación de la propuesta ética desde un criterio

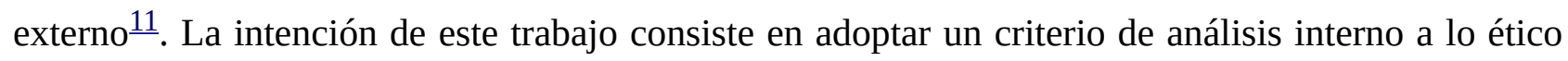
sin recurrir al arbitraje de lo religioso. Se trata, entonces, de leer el proyecto existencial de la segunda parte de $O$ lo uno o lo otro únicamente a partir de sus propias metas. En otras palabras, se trata de exponer el pensamiento del pseudónimo ético a partir de su propio objetivo, a saber, el de construir una teoría de la personalidad capaz de resolver las contradicciones intrínsecas de la vida estética, prescindiendo, en la medida de lo posible, de una fundamentación metafísica (Cfr. Heller, 2008, p. 2).

\section{iii. La desesperación (estética) como índice del carácter auto-relacional de la existencia}

Una de las tesis fundamentales de $\mathrm{B}$, el autor pseudónimo de El equilibrio entre lo estético y lo ético en la formación de la personalidad, es que solo aceptando ciertos aspectos de la visión ética de la vida es posible comprender la verdadera raíz del malestar existencial que agobia a los individuos estéticos $\underline{12}$. El concepto de desesperación (Fortvivlelsen) le permite al individuo ético ver aquello que permanece oculto ante la mirada de los estetas, incluso ante la de aquellos más refinados e intelectuales. Según el pseudónimo ético, en toda interacción del individuo con el mundo se esconde una interacción del individuo con su propio ser. El individuo que se dirige intencionalmente al mundo también se dirige intencionalmente a sí mismo; claro está que, en su 
vida cotidiana, el individuo sólo tiene acceso consciente a su relación con el mundo, mientras que su relación consigo mismo permanece, en primera instancia, sin tematizar. Precisamente es en la elucidación teórica de la experiencia de la desesperación donde queda en evidencia este movimiento doble del individuo, hacia el mundo y hacia sí mismo. Clarifiquemos esta cuestión a través de la refutación ética de la existencia estética.

La esfera estética incluye una amplia y variada gama de prácticas existenciales, que van desde el más craso y vulgar sensualismo hasta una vida consagrada a la delectación de los más altos placeres intelectuales. Se adivina, con todo, que pese a sus notables diferencias estos diversos modos de habitar el mundo pueden ser definidos a través de una misma expresión: el individuo estético tiene como finalidad gozar de la vida $\underline{13}$. En su apetencia de gozo el esteta se entrega a los objetos del mundo y pretende encontrar en ellos la satisfacción de sus anhelos. ¿Qué es lo que ocurre cuando la realidad le da la espalda y lo decepciona al no cumplir con sus expectativas? Desespera. Ahora bien, a B le interesa indicar que esta experiencia negativa es, en realidad, el signo visible de un malestar más profundo y oculto $\underline{14}$ :

Veamos entonces por qué desesperan; es que descubrieron haber fundado su vida en algo efímero; pero ¿es esa una razón para desesperar? ¿Ha habido algún cambio esencial en aquello en lo que fundaron su vida?... No ha habido nada nuevo que pudiera ser motivo de un cambio. Si desesperan, por tanto, debe ser porque de antemano estaban desesperados. La única diferencia es que no lo sabían, y esa es una diferencia totalmente fortuita. Se ve entonces que toda concepción estética de la vida es desesperación, y que todo aquel que vive de manera estética está desesperado, lo sepa o no lo sepa. [Y aquí B finaliza su argumento realizando una importante afirmación] Pero cuando se sabe,... la exigencia de una forma superior de existencia es irrecusable (SKS 3, p. 186 / 2006, p. 177).

La mirada del ético va más allá de la mirada del estético: el problema no radica en el hecho de que el individuo fracasa en su relación con el mundo; sino en el hecho de que el individuo fracasa en su relación consigo mismo. Toda vida estética es, en el fondo, desesperación. ¿Por qué motivo? Porque todos los estetas ponen el centro de gravedad de su existencia por fuera de sí mismos: "aquel que dice querer gozar de la vida -explica B- pone siempre una condición, la cual puede ser exterior al individuo, o bien estar en el individuo de manera tal que no se encuentra en él” (SKS 3, p. 175 / 2006, p. 167). Esta diferenciación que traza el pseudónimo ético, y que tiene como objetivo hacer justicia a las distintas clases de esteticismo, es fundamental. El hedonista irreflexivo aparece dominado completamente por las cosas, tanto su ser como su obrar en el mundo están determinados por el objeto de su deseo. Se entiende, sin dificultad, por qué este tipo de individuo estético pone la condición de su existencia por fuera de sí mismo. Para salvar las consecuencias negativas de la anterior situación, el esteta reflexivo ya no se complace en la posesión del objeto deseado sino que opta por gozar del deseo mismo. El accionar del esteta reflexivo, por tanto, da cuenta de una primera comprensión del hecho de que en toda relación del individuo con el mundo está en juego una relación del individuo consigo mismo. Sin embargo, esta primera comprensión es parcial e incorrecta. A pesar de que el esteta reflexivo cree estar poniendo en sí mismo la condición de su goce, en realidad recae en el mismo inconveniente que busca superar: al depender del deseo, su proyecto vital vuelve a quedar en manos de un factor que excede el control de su voluntad (Cfr. 
SKS 3, p. 184 - 185 / 2006, p. 176). Este traspaso del esteta irreflexivo al esteta reflexivo -un pasaje de figuras de la conciencia que es todavía inmanente al plano estético (Cfr. Milles, 2008, pp. 165 166) - es leído por B como un acercamiento a su propia posición. Son las formas superiores de la existencia estética las que ya apuntan en dirección a la necesidad de desplazar el centro de gravedad de la existencia desde el exterior (el mundo) al interior (el sí mismo). Pero con ello es la misma estética la que internamente se orienta a su superación en la ética ${ }^{15}$.

\section{iv. La «amistad consigo mismo» como condición de posibilidad de una existencia lograda}

Si se le pidiese al pseudónimo ético que defina lacónicamente la relación que el individuo estético tiene consigo mismo la expresión elegida sería, probablemente, “indiferencia” o “desinterés”. Sería erróneo pensar que el pseudónimo ético le recrimina a su interlocutor cierta apatía o descuido en lo que respecta a sus objetivos vitales. Que el esteta desespere en un sentido ordinario es señal de que a él le concierne el resultado de su interacción con el mundo. Sale al paso, entonces, una posible objeción: ¿no es esta misma preocupación del individuo estético por el resultado de sus acciones un signo incontrastable de que él, de hecho, se preocupa por sí mismo? En efecto lo es y ello nos obliga a precisar cuál es el sentido específico en el que de acuerdo con B el individuo estético no se interesa por sí mismo.

La preocupación del individuo por el resultado de su accionar en el mundo denota un primer nivel de preocupación por sí mismo. Para que este actuar conforme a fines sea exitoso es necesario que el individuo posea cierto grado de conocimiento y organización de sí mismo. El individuo debe saber qué es lo que quiere y, al mismo tiempo, tiene que estructurar sus esfuerzos para obtenerlo. Cuanto mayor es la conciencia de los propios deseos y mayor es la capacidad de coordinación de las propias fuerzas, mayores son también, por ende, las chances de que el individuo alcance sus metas. Es, por tanto, la misma referencia al mundo la que requiere y promueve el trato del individuo consigo mismo. B está dispuesto a aceptar que este primer nivel de auto-relación está presente en la vida estética, pero si él le reprocha algo al existente estético es que este sea incapaz de trascender este registro.

En este nivel primario hay una marcada preeminencia del mundo que determina al individuo a asumir una posición instrumental consigo mismo. Dicho con las palabras de B, como la condición del gozo está fuera del individuo, este debe constituirse a sí mismo en réplica a las exigencias de esa exterioridad. Pero esta preponderancia del mundo genera una distorsión en la relación del esteta consigo mismo. Para ilustrar esta afirmación es útil prestarle atención a un pasaje del escrito pseudónimo en el cual se ofrece una descripción de la situación vital del esteticista. B escribe que el alma del esteta "es como un terreno en que crece todo tipo de hierbas, y en el que todas por igual reclaman que se las cultive; su sí mismo consiste en esa multiplicidad, y no tiene ningún "sí mismo» que sea superior a ese” (SKS 3, p. 215 - 216 / 2006, p. 204). Este pasaje nos permite terminar de comprender en qué consiste la "indiferencia” del esteta. El esteta se comporta pasivamente frente a los elementos que conforman su personalidad. El individuo estético quiere, desde ya, satisfacer los deseos que tiene, y quiere, también, realizar sus fines; pero no se pregunta ni si quiere tener esos deseos ni cómo se han determinado sus fines. Acepta que estos elementos simplemente están allí. Las cuestiones que influyen sobremanera en su propia existencia parecen no 
importarle; se comporta ante la riqueza de su vida interior de la misma manera en que se comporta con los objetos del mundo, es decir, se auto-cosifica.

La teoría ética desarrollada en la segunda parte de $O$ lo uno o lo otro busca resolver esta situación. Según la propuesta de B, la relación del individuo consigo mismo debe adquirir prioridad frente a la relación del individuo con el mundo. B no tiene en mente la determinación del mundo a partir del

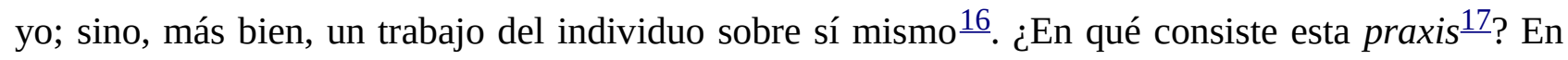
elegirse a sí mismo (vcelge sig selv). ¿Cuál es la idea por detrás de esta célebre y enigmática expresión? Ante todo se trata de dejar atrás el comportamiento auto-cosificador. Eligiéndose a sí mismo el individuo asume ante su propio ser una actitud de implicación existencial y adopta un rol activo frente a la pluralidad de sus vivencias internas: ahora se enfrenta con sus sentimientos, deseos, voliciones e ideas y consiente, rechaza, promueve, anula, discute y corrige estas disposiciones interiores. Esto no significa que a partir de la auto-elección el individuo sea capaz de producir a su antojo sus deseos, sus voliciones, sus sensaciones; pero tampoco se limita a tomar noticias de ellas, sino que al asumir una actitud frente a ellas las hace suyas. En otras palabras, al elegirse a sí mismo el individuo "se posee a sí mismo" (SKS, 3, p. 214 / 2006, p. 202). A través de la auto-elección, el individuo no sólo ejecuta un acto de conocimiento de sí, sino que realiza una comprensión apropiadora: "El individuo ético se conoce a sí mismo, pero ese conocimiento no es una mera contemplación, pues de ese modo el individuo se determina según su necesidad; es un recapacitar sobre sí mismo que es de suyo un actuar” (SKS 3, p. 246 / 2006, p. 232)ํㅗㄹ. De este modo, al posicionarse frente a los elementos de su vida interior, el individuo, en cada una de sus elecciones, va construyendo su identidad. Esta identidad se convierte en el parámetro a partir del cual el individuo quiere definir y evaluar su relación con el mundo; la conformación de esta identidad, en suma, es el movimiento por el cual el individuo sitúa, cada vez más y más, el centro de gravedad de su existencia dentro de sí $\underline{19}$.

Sólo el individuo que se elige correctamente a sí mismo, es decir, el individuo que acepta regir su vida por los principios éticos, establece una relación positiva consigo mismo. En las primeras páginas de su carta, B admite que con su teoría de la auto-elección ética no hace más que exigirle al individuo que se ame a sí mismo (Cfr. SKS 3, p. 165 / 2006, p. 158). El pseudónimo parece descartar rápidamente esta idea; sin embargo, hacia el tramo final de su escrito la recupera. La noción de amor a sí mismo reaparece en un pasaje en el cual el pseudónimo discute su teoría sobre los deberes, un curioso fragmento que contiene expresiones un tanto desconcertantes:

Solo cuando uno considera la vida éticamente, por tanto, alcanza esta su belleza, su verdad, su sentido, su consistencia; solo cuando uno mismo vive de manera ética, solo entonces alcanza la vida de uno su belleza, su verdad, su sentido, su seguridad; solo en la concepción ética de la vida se alivia la duda autopática (autopathiske Tvivl) y la duda simpática (sympathiske Tvivl). Por cierto, la duda autopática y la duda simpática solo pueden aliviarse en un mismo punto, dado que son esencialmente la misma duda. La duda autopática no es, pues, una expresión de egoísmo (Egoisme), sino una exigencia del amor propio (Selvkjerlighed), que es igualmente una exigencia para el propio sí mismo y para el sí mismo de todos los demás. Esto, en mi opinión, es de gran importancia (SKS 3, p. 258 / 2006, p. 242) 
En la primera mitad de este pasaje el pseudónimo insiste sobre el leitmotiv de su escrito: lo ético no anula lo estético. B considera que el modo de vida ético es condición de posibilidad para la consumación de las notas positivas que están presentes en la existencia estética. La vida ética no entraña una rigurosa renuncia al mundo, de hecho B no oculta su enemistad con el ideal de vida ascético del anacoreta o con el estilo de vida del místico (Cfr. SKS 3, p. 229 - 238 / 2006, pp. 216 224). Por el contrario, la existencia ética está guiada por el objetivo de lograr la mayor coincidencia posible entre el deseo y el deber. La oposición irreconciliable entre deseo y deber, argumenta B, es producto de un entendimiento erróneo del concepto de deber (Pligt). Correctamente concebidos los deberes no son imperativos abstractos que se imponen al individuo desde el exterior; son, más bien, una íntima incumbencia ${ }^{\underline{20}}$ que, en la medida en que surgen desde el interior de la personalidad, no suponen una escisión en el yo $\underline{\underline{21}}$.

Luego de esta aclaración inicial, en la segunda parte del fragmento, aparece la noción de amor a sí mismo acompañada por las expresiones “egoísmo”, “duda autopática” y “duda simpática”. Con la expresión “duda simpática” se alude a la preocupación por la personalidad del semejante, se trata de un estar afectivamente interesado en el malestar ajeno. La expresión “duda autopática” se utiliza para mentar la preocupación por la propia personalidad, se trata de un estar afectivamente interesado en el propio malestar. B afirma que esta preocupación de sí no es una manifestación del

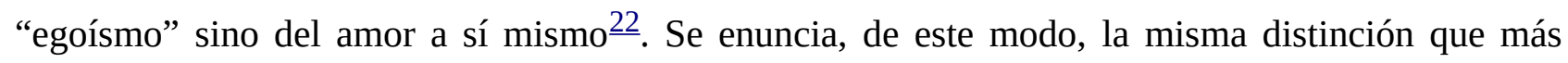
adelante aparecerá en Las obras del amor y La enfermedad mortal: una auto-relación de signo positivo y una auto-relación de signo negativo. Es importante destacar que B le otorga a esta autorelación positiva el rango de exigencia universal, el amarse a sí mismo es para todo hombre un deber. Afirmando que el amor a sí mismo es un deber, el pseudónimo ético da a entender, de modo implícito, que la auto-relación positiva no es algo dado. Amarse a sí mismo exige la transformación de una actitud previa insatisfactoria, que puede asumir o bien la forma de la indiferencia/desinterés de sí o bien la forma de la referencia desmedida a sí mismo. Ahora bien, es de nuestro especial interés recalcar que B considera que esta referencia a sí mismo no sólo es legítima (Cfr. Larrañeta, 1990, p. 206), sino también necesaria para una vida bien lograda o, en sus términos, para la constitución de la personalidad.

Mientras el egoísmo cierra al individuo sobre su personalidad, el amor a sí mismo es un movimiento de auto-remisión y apertura del individuo al mundo:

Si ahora digo que el individuo tiene su teleología en sí mismo, puede que esto dé lugar a un malentendido, como si de esa manera dijese que el individuo sería lo central, o que el individuo en sentido abstracto se bastaría a sí mismo; en efecto, cuando se lo toma de manera abstracta, no obtengo movimiento alguno. El individuo tiene su teleología en sí mismo, tiene teleología interna, es él mismo su teleología; su «sí mismo» es entonces el fin al que aspira. Pero ese sí mismo suyo no es una abstracción, es absolutamente concreto. Al moverse hacia sí mismo, no puede relacionarse de manera negativa con su entorno, pues entonces su sí mismo sería y llegaría a ser una abstracción; es preciso que su sí mismo se abra en pos de su concreción total, pero esa concreción involucra también aquellos factores orientados a intervenir de modo efectivo en el mundo. Así, pues, el suyo es un movimiento a partir de sí mismo hacia sí mismo a través del mundo (SKS 3, p. 260 - 261 / 2006, pp. 244 $-245)$ 
Solo quien se relaciona positivamente consigo mismo puede establecer una relación positiva con su entorno. El pseudónimo ético acepta la tradicional división de los deberes en tres grandes grupos: (1.) hacia Dios, (2.) hacia el mundo y (3.) hacia sí mismǒㅡ. El deber de amor a sí mismo estaría incluido, obviamente, dentro del tercer grupo. No obstante, hacer hincapié en la inclusión del amor a sí mismo dentro de un grupo específico de deberes podría llegar a ocultar el papel central que cumple hacia el interior de la teoría ética de B. En lo que se refiere a la determinación de los deberes el amor a sí mismo cumple una función principal: permite la mediación entre los deberes del individuo consigo mismo y el resto de sus deberes. En otras palabras, es el deber central que permite la deducción de los restantes deberes.

A toda teoría de los deberes le sale al paso una objeción fundamental. Del individuo, nos dice B, se espera que realice lo universal; no obstante, siendo él justamente un individuo, sólo puede realizar lo particular. El único modo de resolver esta cuestión es admitir que el individuo únicamente puede cumplir con el deber cumpliendo con su deber: "Nunca digo que un hombre cumple con el deber o con los deberes, sino que cumple con su deber; digo que yo cumplo con mi deber, y que tú cumples tu deber” (SKS 3, p. 251 / 2006, p. 236). En tanto y en cuanto la teoría ética es una teoría es, necesariamente, general, y por ello sólo le dice al individuo que tiene deberes, pero no le dice -ni puede decirle- cuáles son esos deberes $\underline{24}$. Nuestra hipótesis es que, precisamente, el amor a sí mismo es lo que posibilita la transición de lo general a lo particular; solo el amor a sí mismo permite la singularización de lo universal. ¿Cómo conozco mis deberes? comprendiéndome a mí mismo, adentrándome cada vez más y más en mi propia identidad, es decir, relacionándome positivamente conmigo mismo. Sólo cuando me intereso efectivamente en mi propia personalidad puedo saber cuáles son mis deberes, y sólo conociendo mis deberes puedo relacionarme positivamente con el mundo. El amor a sí mismo, por tanto, es el pilar sobre el cual se construye la unificación entre el deseo y el deber a la cual aspira la existencia ética.

Quien se ama a sí mismo se esfuerza por unificar, integrar y cultivar los componentes de su propia personalidad: "Se tiene a sí mismo como tarea, entonces, de modo tal que esta consiste sobre todo en ordenar, formar, atemperar, incitar, reprimir, en suma, en aportar al alma una equidad, una armonía que es el fruto de las virtudes personales” (SKS 3, p. 250 / 2006, p. 235). Entendida de este modo, la noción de amor a sí mismo del pseudónimo ético se acerca a una importante noción de la ética de Aristóteles. Si mencionamos la proximidad con la ética aristotélica, lo hacemos porque consideramos que la expresión elegida por el filósofo griego envuelve una clara ventaja por sobre la expresión empleada por el pseudónimo ético. En el capítulo 4 del Libro IX de la Ética Nicomáquea, el discurso de Aristóteles limpia el concepto de una auto-relación positiva de sus connotaciones negativas: en lugar de la fórmula amante de sí mismo, el filósofo griego se inclina por la fórmula amigo de sí mismo. Pero ¿es posible utilizar la fórmula aristotélica para describir lo que B tiene en mente?

Cuando Aristóteles habla de «amistad consigo mismo» lo hace teniendo en mente un concepto depurado de amistad. Propiamente hablando, amigos no son aquellos que traban entre sí relaciones de asistencia material recíproca o quienes comparten el tiempo únicamente motivados por afinidades emocionales o intelectuales. Dos individuos son auténticamente amigos si cada uno de ellos está esencialmente interesado en la promoción del carácter virtuoso del otro. La amistad 
consigo mismo, como vemos, nada tiene que ver con la auto-indulgencia o la auto-complacencia. Amigo de sí mismo es quien se preocupa por alcanzar un equilibrio afectivo interior, es quien está interesado en adquirir una unidad de carácter (una identidad volitiva) y quien promueve el desarrollo armónico de sus propias virtudes $\underline{25}$. Un individuo llega a ser amigo de sí mismo cuando encuentra un polo orientativo para sus acciones, un plan o proyecto de vida que sirve como criterio normativo a partir del cual es posible evaluar el curso concreto de su propia existencia. Esta última idea no es para nada ajena a la teoría de la existencia de B, en general, y a su teoría de la amistad, en particular $\underline{26}$. Las relaciones entre amigos, dice el pseudónimo ético, no deben fundarse en el cálculo utilitario; por otra parte, tampoco alcanza la mera simpatía como fundamento de la verdadera amistad (Cfr. SKS 3, p. 301 / 2006, p. 280 - 281). "La condición absoluta de la amistad -[escribe B] es la unidad en la concepción de la vida” (SKS 3, p. 301 / 2006, p. 281). Tener una concepción de la vida no significa simplemente tener una idea o visión sobre el significado y finalidad de la vida (Cfr. SKS 3, p. 175 / 2006, p. 167); se posee realmente una concepción de la vida cuando la propia existencia del individuo deja traslucir el esfuerzo que este realiza para vivir en conformidad con su visión de la vida. La amistad, dice explícitamente B, “contribuye a que uno acceda a la realidad de manera ética” (SKS 3, p. 304 / 2006, p. 283). ¿Por qué? Los amigos coinciden en la idea o visión que ellos mismos se forman sobre la existencia; ellos comparten los mismos compromisos y convicciones éticas y, por eso, se asisten el uno al otro en la realización y consolidación de estos compromisos y convicciones $\frac{27}{}$. Los amigos se exhortan mutuamente a sostener una vida ética.

Hablar de amistad con uno mismo, en nuestra opinión, permite sostener la propuesta del pseudónimo ético sin dejar de hacer justicia a la complejidad de la vida interior del ser humano, una vida interior caracterizada por una pluralidad de vivencias no siempre exenta de desacuerdos y oposiciones. La expresión amigo de sí mismo hace referencia a un desdoblamiento de la personalidad que le permite al individuo entablar una relación pedagógica consigo mismo. Amigo de sí mismo, entonces, es aquel que procura unificar su querer y actuar, aquel que se exhorta a sí mismo para que las distintas manifestaciones de su voluntad y su deseo compartan una misma orientación, una misma concepción de la vida.

\section{v. Consideraciones finales:}

O lo uno o lo otro II define la tarea ética-existencial del individuo como un arduo itinerario de apropiación de sí. Utilizando una expresión de cuño freudiano, se trata de un proceso cuyo objetivo constante es el robustecimiento del yo. La teoría ética de la personalidad, como vimos, afirma que una identidad personal bien lograda no puede forjarse como mera réplica de las exigencias del mundo, sino que debe surgir a partir de un compromiso dialógico entre la exterioridad y la interioridad en el cual lo interior tendrá siempre la última palabra. La preeminencia del mundo ante el yo debe ser reemplazada por la preeminencia del yo ante el mundo. Este movimiento hacia el yo es, a su vez, un movimiento hacia la libertad.

Para Kierkegaard la cuestión en la cual se dirime la viabilidad del proyecto de su pseudónimo ético se juega en una pregunta muy simple: ¿cómo es posible este movimiento hacia el yo, es decir, hacia la apropiación de la libertad? En este sentido, B sólo es capaz de responder este interrogante presuponiendo un interés existencial del individuo en sí mismo: hay un impulso interior que activa 
aquella actividad individual que tiene como meta la integración de la personalidad. Este presupuesto es el que, por otra parte, le garantiza a B que estén dadas las condiciones de posibilidad para superar la existencia desesperada. Precisamente, estos dos supuestos serán puestos bajo sospecha en las sucesivas obras del danés. En primer lugar, la noción de pecado original desarrollada en El concepto de la angustia viene a complejizar el concepto de libertad: la del hombre es, desde su origen, una voluntad alienada, una libertad trabada, que no puede en su accionar histórico más que reproducir las condiciones de su alienación. En segundo lugar, y en clara referencia a lo anterior, es el impulso que orienta al hombre hacia la constitución de una libertad genuina. Por este motivo, tanto en La enfermedad mortal como en Ejercitación del cristianismo, la identidad auténtica solo se logra en un diálogo con el Otro divino.

\section{Notas}

1 Para las citas de Kierkegaard utilizamos la última edición de sus obras completas indicando en números arábigos tanto el volumen como la paginación: Søren Kierkegaard Skrifter (SKS), Copenhague, Gad, 1997 - 2009. Ofrecemos también la paginación correspondiente de las traducciones al castellano consignadas en la Bibliografía.

$\underline{2}$ El trasfondo de la crítica de Adorno es, en este caso, el análisis freudiano del imperativo de amor al prójimo desarrollado en el capítulo V de El malestar en la cultura (1930).

$\underline{3}$ Es difícil decidir si el fenómeno de condicionalidad opera también en sentido inverso, es decir, si la relación del yo con el otro condiciona la relación del yo consigo mismo. En todo caso, podría decirse que de entre todas las relaciones del yo con sus otros existe una relación en la cual la relación del yo con el otro tiene preeminencia por sobre la relación del yo consigo mismo, a saber, en el vínculo entre el individuo y Dios.

4 Sobre el hecho de que toda relación con el otro implica, de suyo, una auto-relación: cfr. GrØn (2005) y Stokes (2003).

$\underline{5}$ “No obstante, en ningún momento introduce la hipótesis que suponga que el hombre sea capaz de no amarse a sí mismo, o incluso de odiarse y destruirse. Si bien se analiza el modo en que generalmente el ser humano se ama a sí mismo, egoístamente, no se discute la posibilidad de que este fenómeno no se produzca” (Dip, 2012, p. 75). Existen, no obstante, en Las obras del amor, algunos pasajes en los cuales se vislumbra cierta auto-negación del sí mismo: “¿Y cuál otro es el pecado de un hombre, que trágicamente opina prestar un servicio a Dios martirizándose a sí mismo, sino el de no querer de veras amarse a sí mismo? ¡Ay!, y quien atenta contra su vida, ¿no peca precisamente en cuanto no se ama con rectitud a sí mismo en el sentido en que un hombre debe amarse?” (SKS 9, p. 30 / 1965, p. 72).

6 Esta cuestión ya había sido señalada por el pseudónimo Vigilius Haufniensis (El concepto de la angustia, 1844) en su crítica al concepto idealista de auto-conciencia. Cfr. "El contenido más concreto de una conciencia es cabalmente la conciencia de sí, la conciencia del individuo mismo. Esta no es la conciencia del yo puro, sino simplemente la conciencia del propio yo... Esta conciencia del propio yo no es una mera contemplación. Quien crea tal cosa muestra bien a las 
claras que no se ha entendido a sí mismo ¿No ha comprobado acaso que él mismo está, simultáneamente, en trance de devenir? ¿Cómo iba a ser entonces un objeto acabado de la contemplación? Por lo tanto, esta conciencia del yo es un acto, y este acto es a su vez interioridad” (SKS 4, p. 443 / 1984a, p. 178).

$\underline{7}$ “La desesperación aparece cuando el sí mismo en la debilidad no quiere ser sí mismo, pero también aparece cuando el sí mismo desafiante quiere ser sí mismo... De cualquier modo, el sí mismo no acepta la tarea que le ha sido dada a su persona por Dios. De modo similar, en Las obras del amor vemos que el sí mismo puede evitar el amor a sí mismo propio amándose a sí mismo en un modo egoísta y pecaminoso; lo que equivale a la desesperación desafío de querer ser sí mismo. O, el sí mismo puede evitar el amor a sí mismo propio no queriendo amarse propiamente; lo que equivale a la desesperación debilidad de no querer devenir sí mismo” (Gregor, 2008, p. 927 nota al pie).

$\underline{8}$ Si bien fácticamente el yo es capaz de rechazarse a sí mismo, para Anti-Climacus este posicionamiento negativo no puede dejar de ser un querer auto-referido del yo. Este es, entre otros, uno de los motivos por los cuales Anti-Climacus (cfr. SKS 11, p. 130 / 1984b, p. 36) entiende que la desesperación debilidad (no querer ser sí mismo) es, en definitiva, una modalidad de la desesperación desafío (querer ser sí mismo).

9 Cfr. "la fórmula que describe la situación del yo una vez que ha quedado exterminada por completo la desesperación es la siguiente: que al auto-relacionarse y querer ser sí mismo, el yo se apoye de una manera transparente en el Poder que lo ha creado” (SKS 11, p. 130 / 1984b, p. 37). Donde optamos por el término transparente (también podría ser traslúcida), Demetrio Rivero elige lúcida. El término danés es gjennemsigtigt, que literalmente significa “ver a través”. El término transparente es mejor porque, a diferencia de la palabra lúcida, es neutral desde un punto de vista cognitivo.

10 La última y verdadera alternativa planteada por Kierkegaard no es entre lo estético y lo ético, sino entre lo mundano (estética y ética) y lo religioso (cristianismo).

11 Puede detectarse, ciertamente, dentro de la exposición del pseudónimo B, una crítica inmanente, es decir, una crítica ética de la ética. No obstante, estas observaciones no implican, para B, el colapso interno de su concepción vital. La crítica ética de la ética, más bien, llama la atención sobre posibles desviaciones internas, pero no alcanza a detectar un déficit constitutivo dentro del planteamiento de B.

$\underline{12}$ B señala que A carece de una auténtica concepción de la vida (cfr. SKS 3, p. 195 / 2006, p. 185). Muñoz Fonnegra, asumiendo la perspectiva de B, señala lo siguiente: “es importante resaltar que lo estético entendido como forma de existencia, como concepto de vida, solo se puede definir y caracterizar desde un nivel cualitativo más alto, esto es, desde lo ético. De acuerdo con la estrategia argumentativa de Kierkegaard, toda comprensión de lo estético desde lo estético se sustrae a su esencia, es decir, es incapaz de asir su elemento más íntimo, debido a que la existencia estética está dispersa en una multiplicidad de instantes que carecen de conexión alguna (2008, p. 843)”.

$\underline{13}$ Cfr. SKS 3, p. 175 / 2006, p. 167. Existen dos traducciones al castellano de la segunda carta de B. La primera de ellas publicada en Buenos Aires en el año 1959 por la Editorial Nova, que contaba 
con una traducción a cargo de Marot. El problema de esta traducción es que utiliza como fuente una versión francesa. La segunda traducción, que utilizamos en nuestro trabajo, es de Darío González, que ha trabajado con el texto fijado por la última edición de las obras completas del danés.

14 Para un análisis del concepto de desesperación en $O$ lo uno o lo otro y sus transformaciones en La enfermedad mortal puede consultarse Kosch (2006).

15 Resulta claro que esta tendencia interior no opera en el plano del pensamiento y la acción deliberada del individuo estético. Claramente estamos aquí ante un procedimiento dialéctico de corte hegeliano. El individuo estético sólo puede realizar su proyecto vital deviniendo individuo ético; él quiere a través de su proceder permanecer en el plano estético, pero ese mismo proceder lo pone en el límite de lo ético.

16 Esta opción radical debe estar a la base de cualquier intento de construcción de una propuesta política a partir de Kierkegaard. En el plano de la transformación social, Kierkegaard, como supo señalar Agnes Heller, se constituye en una alternativa excluyente frente a la solución marxista: “Los filósofos hasta ahora solo han explicado el mundo, declaran tanto Kierkegaard como Marx. Pero uno dice: es preciso cambiar nuestra relación con el mundo; y el otro: es preciso cambiar al mundo mismo" (Heller, 1999, p. 177).

$\underline{17}$ Utilizamos la palabra praxis en el sentido que le asigna Aristóteles en su Ética Nicomáquea, es decir, en el sentido de una acción que tiene como fin la transformación del agente para distinguir esta actividad de una que tiene como meta la producción de un objeto externo: "El fin de la producción [poiesis] es distinto de ella, pero el de la acción [praxis] no puede serlo; pues una acción bien hecha es ella misma el fin (VI, 5, 1140b 5 - 8)”.

18 Sobre esta misma idea volverá Kierkegaard en El concepto de la angustia de 1844: "El contenido más concreto de una conciencia es cabalmente la conciencia de sí, la conciencia del individuo mismo. Esta no es la conciencia del yo puro, sino simplemente la conciencia del propio yo... Esta conciencia del propio yo no es una mera contemplación. Quien crea tal cosa muestra bien a las claras que no se ha entendido a sí mismo ¿No ha comprobado acaso que él mismo está, simultáneamente, en trance de devenir? ¿Cómo iba a ser entonces un objeto acabado de la contemplación? Por lo tanto, esta conciencia del yo es un acto, y este acto es a su vez interioridad” (SKS 4, p. 443 / 1984a, p. 178).

19 Cfr. "Sólo en sí mismo tiene el individuo la meta a la que debe aspirar... Cuando se ha conocido a sí mismo y se ha elegido a sí mismo, el individuo está realizándose a sí mismo, pero, puesto que debe realizarse a sí mismo libremente, debe saber qué quiere realizar. Es cierto que eso que el individuo quiere realizar es él mismo, pero es su sí mismo ideal, el cual, sin embargo, no puede hallar en otra parte que en sí mismo” (SKS 3, p. 247 / 2006, p. 232.)

$\underline{20}$ La palabra danesa Pligt deriva del verbo at pleje que puede traducirse al castellano como "cuidar" o "tomar a cargo".

21 Cfr. "el deber no es una añadidura, sino una incumbencia. Cuando se lo ve de ese modo, el deber es una señal de que el individuo está orientado en sí mismo... Cuanto más profundamente ha cimentado el hombre su vida de manera ética, tanto menos sentirá la necesidad de hablar del deber a cada instante, de angustiarse a cada instante respecto de si lo ha cumplido, de consultar a cada 
instante a los demás respecto de cuál es su deber. Visto de la manera correcta, lo ético da al individuo una infinita seguridad en sí mismo; visto de modo incorrecto, da inseguridad al individuo, y no puedo imaginarme una existencia más desdichada o más atormentada que la de un hombre que, teniendo el deber fuera de sí mismo, quiere continuamente realizarlo” (SKS 3, pp. 242 - 243 / 2006, pp. 228 - 229).

22 "Una existencia ética es una existencia preocupada o 'interesada'. Esto no es egotismo (Egoísmo), sino yo-idad o subjetividad (Egoidad). Existir en la interioridad subjetiva como un sí mismo no es una inflamación narcisista del ego, sino una tarea ética, pero una tarea ética que requiere auto-control, auto-dominio, auto-disciplina, un tipo de ascetismo espiritual” (Stack, 1973, p. 112).

$\underline{23}$ Cfr. SKS 3, p. 253 / OO II, p. 238. Esta clasificación ya aparece en La metafísica de las costumbres de Kant y en el antecedente directo de B, el catecismo de Balle. Para un análisis de la relación entre B y Balle puede consultarse Watkin (1995).

$\underline{24}$ Con todo una teoría ética puede formular deberes precisos, pero en ese caso estaría equivocando el camino porque establecería una relación conflictiva entre el individuo y el deber: "Puede que a uno le parezca haber eludido todo escepticismo [B se refiere con este término a la objeción anteriormente aludida] haciendo del deber algo exterior, fijo y determinado, algo que lo que podría decirse: eso es el deber. Pero ese es un malentendido, pues la duda no reside en lo exterior sino en lo interior, en mi relación con lo general” (SKS 3, p. 251 / 2006, p. 236).

$\underline{25}$ “Este, en efecto, está de acuerdo consigo mismo y desea las mismas cosas con toda su alma, y quiere y practica para sí el bien y lo que parece así (pues es propio del hombre bueno trabajar con empeño por el bien)..." (EN IX 4, 1166a 13 - 16).

26 Cabe señalar que el mismo B reconoce su deuda con Aristóteles en lo que respecta a su teoría de la amistad (Cfr. SKS 3, p. 304 / 2006, p. 283).

$\underline{27}$ “Los amigos -explica Brian Gregor- están unidos por sus compromisos y convicciones éticas. En consecuencia, la amistad es vital porque los amigos se ayudan mutuamente en la actualización y el mantenimiento de esos compromisos” (2008, p. 923).

Por este motivo, para B la verdadera amistad precisa de cierta maduración personal: "Las naturalezas más versátiles se ajustan a sí mismas sin dificultad, su sí mismo es ya desde el comienzo una moneda corriente, y así entablan la transacción llamada amistad. A las naturalezas más profundas no les es tan fácil hallarse a sí mismas, y hasta que no hayan hallado su «sí mismo» no podrán desear que alguien les ofrezca una amistad que no podrían retribuir” (SKS 3, p. 299 / 2006, p. 279). El yo sólo puede entrar en una relación positiva con el otro una vez que se relaciona positivamente consigo mismo; pero, por lo menos si nos movemos dentro del plano inmanente de lo estrictamente humano, esta auto-relación positiva no la logra el yo a través de la relación positiva con los otros. En otras palabras, la amistad (tomada aquí como sinónimo de la relación positiva con el otro) cumple su rol una vez que el individuo se ha singularizado, pero no interviene en el proceso de singularización. 


\section{Bibliografía}

Adorno T. (1969). Kierkegaard. La construcción de lo estético (trad. Vernengo). Venezuela: Monte Ávila Editores.

Aristóteles (trad. en 2000). Ética Nicomáquea (trad. Palli Bonet). Barcelona: Gredos.

Dip, P. (2012). Teoría y praxis en Las obras del amor. Un recorrido por la erótica kierkegaardiana. Buenos Aires: Editorial Gorla.

Gregor, B. (2008). Friends and Neighbors: Kierkegaard and the possibility of transformative Friendship. Revista portuguesa de filosofía, 64(2-4), 921 - 941.

GrØn, A. (2005). Reconocimiento y comunicación. La ética entre Hegel y Kierkegaard. Estudios de Filosofía, 32, 27 - 40.

Heller, A. (1999). Crítica de la Ilustración. Las antinomias morales de la razón (trad. López Soria). Barcelona: Península.

Heller, A. (2008). “The Papers of B as the Modern Answer to both Aristotle and Kant”. En Kierkegaard Studies. Yearbook 2008. Berlin - Nueva York: SØren Kierkegaard Research Center \& Walter de Gruyter.

Kierkegaard, S. (1965). Las obras del amor. Tomo I y II (trad. Rivero). Madrid: Guadarrama.

Kierkegaard, S. (1984a). El concepto de la angustia (trad. Rivero). Madrid: Orbis.

Kierkegaard, S. (1984b). La enfermedad mortal (trad. Rivero). Madrid: Sarpe.

Kierkegaard, S. (2006). O lo uno o lo otro. Un fragmento de vida II (trad. González). Madrid: Trotta.

Kosch, M. (2006). "Despair” in Kierkegaard's Either/Or. Journal of the History of Philosophy, 44(1), $85-97$.

Larrañeta, R. (1990). La interioridad apasionada. Verdad y amor en SØren Kierkegaard. Salamanca: Universidad Pontificia Editorial San Esteban.

Milles, T. (2008). Eihter / Or: Reintroducing an Ancient Approach to Ethics. En Kierkegaard Studies. Yearbook 2008. Berlin - Nueva York: SØren Kierkegaard Research Center \& Walter de Gruyter.

Muñoz Fonnegra, S. (2008). La Ética en la formación de la personalidad. Revista portuguesa de filosofía, 64(2-4), 841 - 857.

Stack, G. (1973). Kierkegaard: the self and ethical existence. Ethics, 83(2), 108 - 125.

Stokes, P. (2003). Kierkegaardian vision and the concrete other. Continental Philosophy Review, 39, $393-413$.

Watkin, J. (1995). Judge William - A Christian?. En Perkins R. (Ed.), International Kierkegaard Commentary. Either / Or. Part II. Volume 4 (pp. 113 - 124). Georgia: Mercer University Press, 1995. 